\title{
Magnetic Microstructure of Magnetotactic Bacteria by Electron Holography
}

\author{
Rafal E. Dunin-Borkowski, Martha R. McCartney, \\ Richard B. Frankel, Dennis A. Bazylinski, Mihály Pósfai, \\ Peter R. Buseck
}

\begin{abstract}
Off-axis electron holography in the transmission electron microscope was used to correlate the physical and magnetic microstructure of magnetite nanocrystals in magnetotactic bacteria. The magnetite crystals were all single magnetic domains, and the magnetization directions of small superparamagnetic crystals were constrained by magnetic interactions with larger crystals in the chains. Shape anisotropy was found to dominate magnetocrystalline anisotropy in elongated crystals. A coercive field between 300 and 450 oersted was determined for one chain.
\end{abstract}

Magnetic crystals below $100 \mathrm{~nm}$ in size occur in organisms in many biological phyla (1). For example, magnetotactic bacteria contain magnetosomes, which are intracellular, ferrimagnetic crystals of magnetite $\left(\mathrm{Fe}_{3} \mathrm{O}_{4}\right)$ or greigite $\left(\mathrm{Fe}_{3} \mathrm{~S}_{4}\right)$. The magnetosomes are usually arranged in one or more linear chains within each bacterium and impart a permanent magnetic moment to the cell that results in its alignment and motion parallel to geomagnetic field lines (2). This behavior is thought to increase the efficiency with which such bacteria find their optimal oxygen concentrations or redox potentials at sedimentwater interfaces or in water columns (3). The crystals in magnetotactic bacteria have specific morphologies within each cell type, and their magnetic easy axes are usually aligned along the chain axis (4). The small sizes of the crystals (typically between 40 and 100 $\mathrm{nm}$ ) suggest that they should each contain a single magnetic domain (5). However, the magnetic microstructure of the magnetosome chain is not completely understood. This information is crucial for understanding the function of biogenic magnetic crystals in magnetic field sensing in higher organisms (6). It is also of interest for the potential applications of single-domain magnets in fields such as electronics, catalysis, and magnetic recording (7). Magnetotactic bacteria provide an ideal system for studying interact-

R. E. Dunin-Borkowski and M. R. McCartney, Center for Solid State Science, Arizona State University, Tempe, AZ 85287-1704, USA. R. B. Frankel, Department of Physics, California Polytechnic State University, San Luis Obispo, CA 93407, USA. D. A. Bazylinski, Department of Microbiology, lowa State University, Ames, IA 50011, USA. M. Pósfai and P. R. Buseck, Departments of Geology and Chemistry/Biochemistry, Arizona State University, Tempe, AZ 85287-1404, USA. ing single-domain magnetic crystals.

The magnetic moments of magnetosome chains have been measured both statistically for large numbers of bacteria (8) and by magnetic force microscopy (MFM). However, MFM has poor resolution when applied to intact bacteria, and the results are difficult to quantify (9). Electron holography in the transmission electron microscope (TEM) has been used to provide improved high-resolution information about materials and to analyze magnetic and electric microfields (10). Here, we use off-axis electron holography in the TEM (Fig. 1) to directly measure the local magnetic fields associated with mineral particles in magnetotactic bacteria.

We examined cultured cells of Magnetospirillum magnetotacticum strain MS-1 and the marine vibrioid strain MV-1, prepared by methods described in $(11,12)$ and deposited onto carbon-coated grids. Off-axis electron holograms of individual cells of both strains were recorded at $200 \mathrm{keV}$ with a Philips CM200 field-emission-gun TEM. In addition, elemental maps of strain MV-1 were recorded at $200 \mathrm{keV}$ with a Philips EM430 TEM equipped with a Gatan post-column imaging spectrometer $(13,14)$

Figure 2A shows a TEM bright-field image of a single cell of $M$. magnetotacticum. The magnetite chain is $1200 \mathrm{~nm}$ long and (excluding the smallest outlying crystals at the ends of the chain) contains 22 crystals that have an average length and separation of $\sim 45$ and $\sim 9.5 \mathrm{~nm}$, respectively. Previous studies have shown that the crystals in $M$. magnetotacticum are combinations of the $\{111\}$ (octahedron) and $\{100\}$ (cube) forms, and the [111] magnetic easy axes of the crystals are primarily parallel to the chain axis (15). A prominent defect in the chain, in the form of two small crystals, is arrowed in the figure. The chain is asymmetrical, and larger crystals are at its left end. Electron holograms were obtained by applying $120 \mathrm{~V}$ to an electrostatic biprism wire in the selected-area aperture plane of the microscope, in order to overlap the electron wave that had passed through the sample with one that had passed only through vacuum (Fig. 2B). The amplitude and phase of the sample wave are recorded in the intensity and position of the resulting interference fringes, respectively $(16,17)$.

The phase is sensitive to the in-plane component of the magnetic induction integrated in the incident beam direction and to the mean inner (electric) potential. Neglecting dynamical diffraction effects (18), the phase is given in one dimension by

$$
\begin{aligned}
\phi(x) & =\left(\frac{2 \pi}{\lambda}\right)\left(\frac{E+E_{0}}{E\left(E+2 E_{0}\right.}\right) \int V(x, z) d z \\
& -\left(\frac{e}{\hbar}\right) \iint B_{\perp}(x, z) d x d z
\end{aligned}
$$

where $z$ is the incident beam direction, $x$ is a direction in the plane of the sample, $B_{\perp}$ is the component of the magnetic induction perpendicular to both $x$ and $z, V$ is the mean inner potential of the sample, $\lambda$ is the wavelength, and $E$ and $E_{0}$ are, respectively, the kinetic and rest mass energies of the incident electron (10). In most magnetic samples, the mean inner potential dominates the phase and must be removed to quantify the magnetization. Accordingly, two holograms were obtained from each area of the sample, between which the magnetization of the magnetite chain was reversed by using the magnetic field of the microscope objective lens. The magnetic and mean potential contributions to the phase were then calculated by taking half the difference and half the sum of the phases of the two holograms, respectively $(14,18)$.

Figure 2C shows contours formed from the magnetic contribution to the holographic phase of the cell of $M$. magnetotacticum when it was examined in field-free conditions (that is, in its remanent state). The density of the contours is proportional to the component of the magnetic induction in the plane of the sample integrated in the incident beam direction (18). The holographic images show that the gradient in the field is lowest where the field decreases at the ends of the chain and at gaps between individual magnetosomes. Some chains show a magnetic asymmetry in which the magnetic contribution is stronger on one end of the chain. The magnetic field lines bend in some of the magnetite crystals to minimize their magnetostatic energy, whereas in others their direction differs slightly from that of the chain axis. Small deviations in the positions of the crystals from the chain axis, rather than magnetocrystalline anisotropy, may have a dominant influence on the direction of the field within 
the crystals (see below).

All the crystals we examined are single magnetic domains, which is consistent with the qualitative prediction of a single- to multidomain transition when the particle size is comparable to the domain wall width of $\sim 100 \mathrm{~nm}$ for bulk magnetite (5). The particle size at which this transition occurs has been uncertain (19). Numerical micromagnetic modeling gives a value of $\sim 70 \mathrm{~nm}$ for cubes (20), which is predicted to increase with axial ratio $(20,21)$. Metastable single domains may exist in the multidomain regime (19), particularly in the presence of interactions between closely spaced particles. Examples of such behavior may be provided by the large (100 to $200 \mathrm{~nm}$ ) biogenic magnetite crystals reported by Farina et al. (22), which would lie in the two-domain regime if they were isolated.

Interactions between magnetosomes likely account for the magnetization of the small crystals at the right end of the chain in Fig. 2C. This observation is somewhat surprising as the crystals are less than the 20 to $35 \mathrm{~nm}$ size below which the magnetization direction of magnetite is expected to be thermally unstable, that is, superparamagnetic, at room temperature (19, 21). The alignment of the magnetization of the smallest crystals suggests that thermal fluctuations of the magnetization are overcome by the field of the larger crystals in the chain and not by local magnetostatic interactions between the small crystals themselves. The results also indicate that nonmagnetic precursors, which have been identified in chains of magnetosomes in greigite-producing magnetotactic bacteria (23), either do not form during magnetite synthesis in magnetotactic bacteria or have already transformed to a magnetic form on the sample

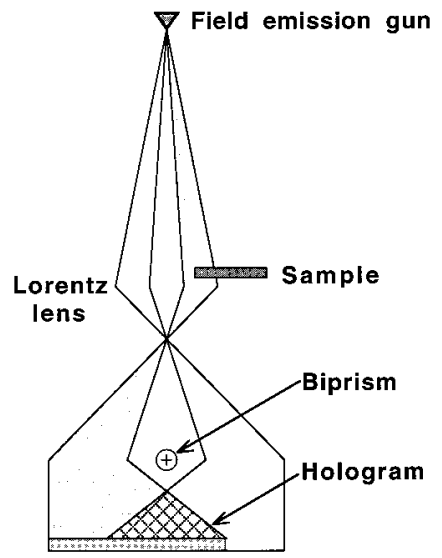

Fig. 1. Schematic illustration of set-up used for generating off-axis electron holograms in the TEM. The sample occupies approximately half the field of view and is coherently illuminated by the field emission electron gun. The positively charged electrostatic biprism (a thin gold-coated quartz fiber, $<0.7 \mu \mathrm{m}$ in diameter) causes overlap of the object and (vacuum) reference waves. The resulting holographic interference pattern is recorded digitally. grid. The two small crystals that form the defect in the chain are magnetized and result in poorer confinement of the field lines but do not significantly decrease the total magnetic dipole moment of the cell.

The magnetic moment of the cell can be determined from the equation

$$
m \approx\left(\frac{\hbar}{e}\right) \int_{\text {chain }}(\Delta \phi) d l
$$

Fig. 2. (A) TEM brightfield image of a single cell of Magnetospirillum magnetotacticum strain MS-1. (B) Off-axis electron hologram obtained in field-free conditions from the region marked in (A). The holographic phase changes can be seen most clearly, in the form of bending of the holographic interference fringes as they pass through the crystals, by looking along the fringes at a shallow angle to the plane of the paper. (C) Contours of spacing 0.064 radians formed from the magnetic contribution to the holographic phase. The contours, which spread out at the ends of the chain, have been overlaid onto the mean
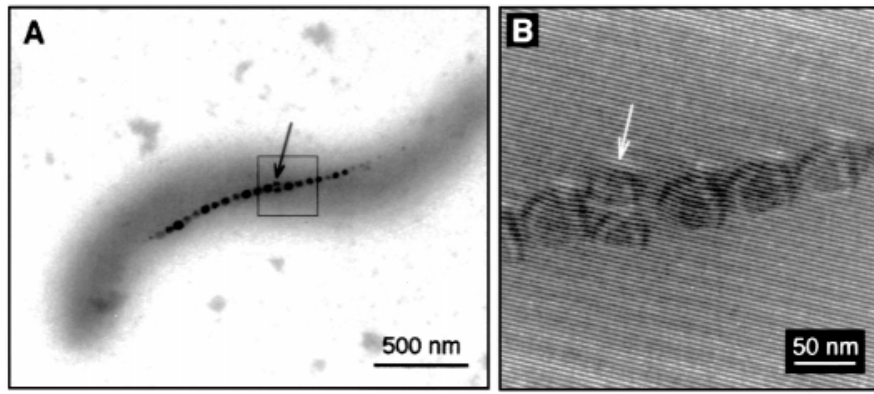

potential contribution

to the phase to allow the positions of the crystals to be correlated with the magnetic contours. It follows from Eq. 1 that the contours are most closely spaced where the projected thicknesses of the crystals are greatest. The intensity of the background differs between the two sides of the chain because of variations in the projected thickness of the cell. The same double crystal defect in the chain is arrowed in each figure.

Fig. 3. (A) As for Fig. 2C but for a single cell of strain MV-1. The contours of spacing 0.064 radians are closest together within the crystals, and they spread out both at the end of the chain and at gaps between individual crystals. (B) TEM bright-field image. (C and D) Threewindow, backgroundsubtracted elemental maps and corresponding line-profiles obtained at $200 \mathrm{kV}$ with a Gatan imaging filter for the $\mathrm{Fe}_{23}$ and $\mathrm{O} \mathrm{K}$ edges, respectively. The

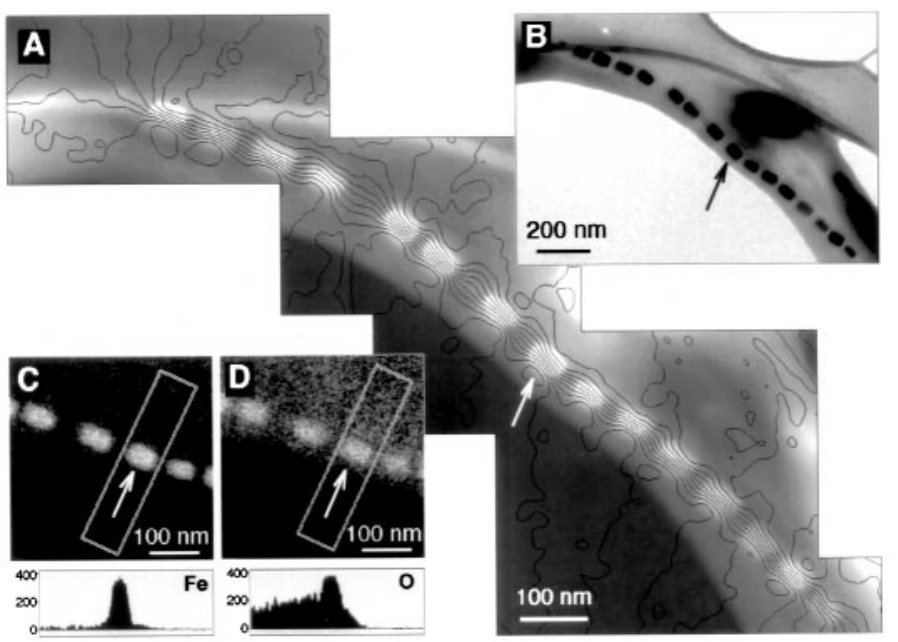
same magnetosome is arrowed in each figure. The noisy background in the oxygen line-profile arises from the organic material around the magnetosomes. where $\Delta \phi$ is the local change in the magnetic contribution to the phase across the chain and $l$ is a direction along the chain axis. A value of $5 \times 10^{-16} \mathrm{~A} \mathrm{~m}^{2}\left(5 \times 10^{-13}\right.$ electromagnetic units) is obtained for the moment of the chain in Fig. 2. This is close to the value calculated by estimating the volume of magin the chain as $2245-\mathrm{nm}$ diamete ignoring the weak demagnetizing field associated with the chain of spheres. This result bulk magnetite of $0.603 \mathrm{~T}\left(480 \mathrm{emu} / \mathrm{cm}^{3}\right)$ and

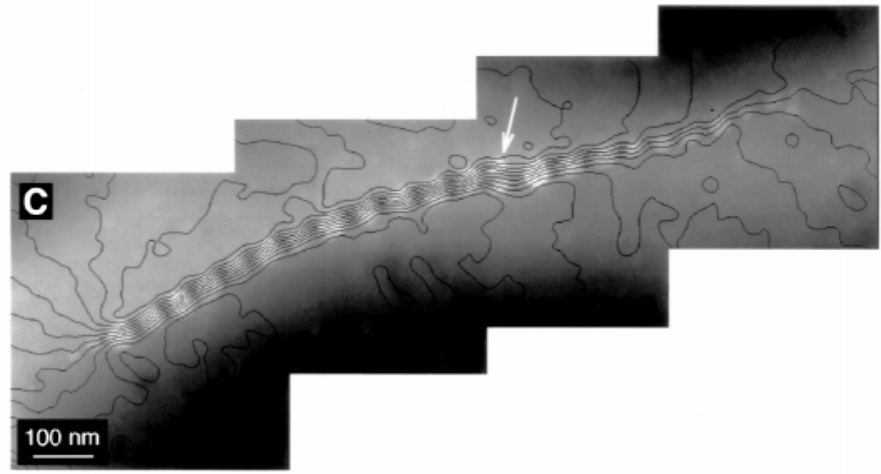


indicates that the remanent magnetization of the chain is the saturation magnetization.

We also examined strain MV-1 (24), which commonly contains large gaps between the magnetosomes (Fig. 3). The crystals in MV-1 are combinations of the $\{110\}$ (dodecahedron) and $\{111\}$ (octahedron) forms and are elongated along [111] (25). The 15 magnetite crystals in the chain have dimensions of $\sim 60 \times \sim 35 \mathrm{~nm}$, and the chain length is $\sim 1600 \mathrm{~nm}$. The magnetic induction drops gradually at the ends of the chain and notably at large gaps between the crystals. The spacings between the crystals are large enough for the chain to resemble a series of solenoids.

The direction of the contours in the fourth magnetosome from the left end of the chain, which is misaligned with respect to its neighbors, shows that the magnetization within elongated crystals is dominated by their shape rather than by the direction of the chain axis. A different behavior is observed for more equiaxed crystal morphologies, as shown in Fig. 4, A and B. In this M. magnetotacticum chain, the morphology of the crystals (Fig. 4A) indicates that they are aligned with their [111] magnetic easy axes along the chain axis, whereas the magnetization direction within two crystals (arrowed) that are offset laterally from the others differs from both their [111] direction and the chain axis (Fig. 4B). The difference between this behavior for MV-1 and M. magnetotacticum is a direct result of the weak magnetocrystalline anisotropy of magnetite (magnetite has a relatively low value for the first anisotropy constant, $K_{1}$, of $1.35 \times 10^{4} \mathrm{~J} \mathrm{~m}^{-3}$, about $30 \%$ of that of $\mathrm{Fe}$ ) and because shape anisotropy of the magnetite crystals plays a dominant role for strain MV-1.

The magnetic moment of the chain in Fig. 3 was measured to be $7 \times 10^{-16} \mathrm{~A} \mathrm{~m}^{2}(7 \times$ $10^{-13} \mathrm{emu}$ ). The moment per unit length is the same as that of the chain in M. magnetotacticum shown in Fig. 2 to within experimental error despite the different crystal mor-

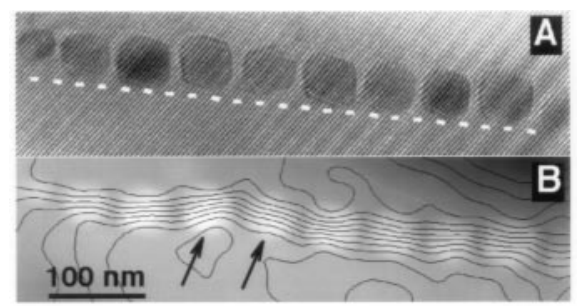

Fig. 4. (A) Electron hologram of part of a magnetosome chain within a cell of M. magnetotacticum strain MS-1. The line in (A) is drawn as a visual aid to show that the two crystals arrowed in (B) are offset slightly from the chain axis. (B) Contours of spacing 0.15 radians formed from the magnetic contribution to the holographic phase overlaid onto the mean potential contribution to phase. phologies in the two cells. Holograms obtained at successive values of the applied magnetic field show that the coercive field of the MV-1 chain is between 300 and 450 Oe. Penninga et al. (26) measured a coercive field of 300 Oe for M. magnetotacticum, although calculations suggest that the exact value for any given chain is sensitive to the particle size, separation, and chain length $(8,27)$, as well as to magnetocrystalline anisotropy. A comparison of our measured coercive field with the results of a chainof-spheres calculation for magnetite (28) shows much better agreement with a symmetric fanning mechanism for reversal than with a mechanism that involves parallel rotation of the moments.

Three-window, background-subtracted elemental maps (29) provide compositional information that can be correlated with the physical and magnetic microstructures. The magnetosomes in the chain within the cell of strain MV-1 are composed of iron and oxygen (Fig. 3, C and D). (The same crystal is arrowed in each image in Fig. 3.) The iron line-profile shows an apparent difference in iron concentration between the two sides of the magnetosome, which is opposite in sense to that in the oxygen profile. This observation could imply that iron is primarily transported through the cell wall adjacent to the magnetosome vesicle and subsequently transformed into the magnetic mineral (for example, magnetite or greigite).

Because superparamagnetic and permanently magnetized fine-grained magnetite can be distinguished with this technique, it could be used to elucidate magnetite-based magnetic sensing mechanisms in higher organisms $(30,31)$. It could also be used to study interparticle interaction effects in high-density recording media and other supramolecular assemblies of ultrafine magnetic particles.

\section{References and Notes}

1. H. A. Lowenstam and S. Weiner, On Biomineralization (Oxford Univ. Press, New York, 1989).

2. R. B. Frankel, Annu. Rev. Biophys. Bioeng. 13, 85 (1984).

3. D. A. Bazylinski, M. S. Johnson, B. L. Taylor, Biophys. J. 73, 994 (1997).

4. D. A. Bazylinski, A. J. Garratt-Reed, R. B. Frankel, Microsc. Res. Tech. 27, 389 (1994)

5. B. M. Moskowitz, Rev. Geophys. Suppl., p. 123.

6. M. M. Walker, C. E. Diebel, C. V. Haugh, P. M. Pankhurst, J. C. Montgomery, Nature 390, 371 (1997); C.-Y Hsu and C.-W. Li, Science 265, 95 (1996); S. Mann, N. H. Sparks, M. M. Walker, J. L. Kirschvink, J. Exp. Biol. 140, 35 (1988)

7. S. Mann, Nature 365, 499 (1993).

8. B. M. Moskowitz, R. B. Frankel, P. Flanders, R. P. Blakemore, B. B. Schwartz, J. Magn. Magn. Mat. 73, 273 (1988).

9. R. B. Proksch et al., Appl. Phys. Lett. 66, 2582 (1995).

10. A. Tonomura, Adv. Phys. 41, 59 (1992); A. Orchowski, W.-D. Rau, H. Lichte, Phys. Rev. Lett. 74, 399 (1995).

11. R. P. Blakemore, D. Maratea, R. S. Wolfe, J. Bacteriol. 140, 720 (1979).

12. D. A. Bazylinski, R. B. Frankel, H. W. Jannasch, Nature 334, 518 (1988).

13. The Philips CM200 TEM was equipped with a rotat- able electrostatic biprism and a 1024 pixel by 1024 pixel charge-coupled device camera. An additional Lorentz minilens (1.2-nm line resolution at $200 \mathrm{keV}$ ), located in the bore of the objective lens pole-piece, allowed samples to be examined in almost field-free conditions with the main objective lens switched off. The objective lens could also be excited slightly and the sample tilted so that magnetization processes could be followed in situ (16).

14. R. E. Dunin-Borkowski, M. R. McCartney, D. J. Smith, S. S. P. Parkin, Ultramicroscopy 74, 61 (1998).

15. S. Mann, R. B. Frankel, R. P. Blakemore, Nature 310, 405 (1984).

16. D. J. Smith and M. R. McCartney, in Introduction to Electron Holography, E. Völkl, L. F. Allard, D. C. Joy, Eds. (Plenum, New York, 1988).

17. The width of the overlap region of the wave that passed through the sample with the wave that passed through vacuum was $640 \mathrm{~nm}$, as referred back to the level of the sample. The spacing of the holographic interference fringes was $3.9 \mathrm{~nm}$. Experimentally, the amplitude and phase of the image were obtained by extracting one "sideband" from the Fourier transform of the hologram. This sideband was then inverse Fourier transformed, and the amplitude and phase of the resulting complex image wave were calculated. Before further analysis, the phase was "unwrapped" to remove the artificial phase discontinuities that result from the fact that it is initially calculated modulo $2 \pi$.

18. The magnetic contribution to the phase was determined from the difference between the phases of two holograms in which the magnetization of the chain was reversed. This procedure also ensures that dynamical contributions from crystals that are diffracting strongly are removed, and justifies the use of Eq. 1 to interpret the results. The spatial resolution of the magnetic information in Fig. $2 \mathrm{C}$ is limited by the 7.8-nm pixel size and the relatively weak magnetic signal. The signal-to-noise characteristics of the hologram limit the resolution for magnetic samples. (Strong, nonmagnetic phase objects allow for spatial resolutions of $0.1 \mathrm{~nm}$.) In general, the magnetic contribution to the phase can be interpreted quantitatively to determine the magnetization of an unknown material whose thickness has been determined independently; however, such an analysis is unwarranted for the present sample without an accurate model for the demagnetizing fields around the crystals.

19. D. J. Dunlop and Ö. Özdemir, Rock Magnetism: Fundamentals and Frontiers (Cambridge Univ. Press, Cambridge, 1997).

20. K. Fabian et al., Geophys. J. Int. 124, 89 (1996).

21. D. A. Bazylinski and B. M. Moskowitz, in Geomicrobiology: Interactions Between Microbes and Minerals, J. F. Banfield and K. H. Nealson, Eds. (Mineralogical Society of America, Washington, DC, 1997), pp. 181223

22. M. Farina, B. Kachar, U. Lins, R. Broderick, H. Lins de Barros, J. Microsc. 173, 1 (1994)

23. M. Pósfai, P. R. Buseck, D. A. Bazylinski, R. B. Frankel, Science 280, 880 (1998)

24. N. H. C. Sparks et al., Earth Planet Sci. Lett. 98, 14 (1990).

25. F. C. Meldrum, S. Mann, B. R. Heywood, R. B. Frankel, D. A. Bazylinski, Proc. R. Soc. London Ser. B 251, 231 (1993).

26. I. Penninga, H. DeWaard, B. M. Moskowitz, D. A. Bazylinski, R. B. Frankel, J. Magn. Magn. Mat. 149, 279 (1995).

27. I. S. Jacobs and C. P. Bean, Phys. Rev. 100, 1060 (1955).

28. P. C. Kuo, J. Appl. Phys. 64, 5071 (1988).

29. R. F. Egerton, Electron Energy-Loss Spectroscopy in the Electron Microscope (Plenum, New York, 1996).

30. J. L. Kirschvink and J. L. Gould, Biosystems 13, 181 (1981).

31. M. Winklhofer et al., Eos 79, S68 (1998).

32. We thank M. R. Scheinfein for discussions. Supported by grants from the NSF and NASA. Electron microscopy was carried out at the Center for High-Resolution Electron Microscopy at Arizona State University. 